\title{
The changing pattern of sexually transmitted disease in adolescent girls
}

\author{
G E ROBINSON, G E FORSTER, AND P E MUNDAY \\ From the Praed Street Clinic, St Mary's Hospital, London W2 INY
}

SUMMARY When girls aged 15-19 attending a sexually transmitted diseases (STDs) clinic in 1972 and 1982 were studied retrospectively, the prevalence of recognised STDs declined both absolutely and as a proportion of the total women studied. The increased number of attendances was accounted for by "other conditions requiring, or not requiring, treatment." An alternative approach for the classification of these conditions is proposed.

\section{Introduction}

In England and Wales physicians in charge of sexually transmitted diseases (STDs) clinics are required to make yearly returns to the Department of Health showing the numbers of new cases with specified STDs. These returns form the basis of statistics that are published yearly. Although attendance at these clinics has increased, the number of cases with some STDs has remained static or even fallen. In the diagnostic category "other conditions", however, the number of cases seen yearly has continued to rise. ${ }^{1}$

We have previously studied the prevalence of STDs in a population of adolescent girls attending the Praed Street Clinic in the years 1972 and 1982. ${ }^{2}$ Although the numbers of young women attending the clinic increased by $31 \%$ (1373 to 1799), most of this increase was explained by attendances for "other conditions" not classified as STD. The number of young women in this group increased by $79 \%$ (613 to 1100 ) between 1972 and 1982 . We attempted to analyse the diagnoses made in this group of adolescents using a problem oriented approach.

\section{Patients and methods}

Using methods described previously, ${ }^{2}$ we compared retrospectively the prevalence of STDs in a population of adolescent girls aged 15-19 who attended the Praed Street Clinic in the years 1972 and 1982. Each patient who had been classified as having "other conditions requiring, or not requiring, treat-

Address for reprints: Dr G E Robinson, Department of Obstetrics and Gynaecology, The London Hospital, Whitechapel, London El

Accepted for publication 11 August 1984 ment" was reclassified in one of seven categories based on the problem with which she presented.

\section{Results}

We studied 1373 patients in 1972, and 1799 in 1982. Further details of the study population have already been presented. ${ }^{2}$ Table I shows that the prevalence of all STDs declined from $80 \%(1094 / 1373)$ in 1972 to $56 \%(1008 / 1799)$ in 1982 despite the increase in clinic attendance during the decade.

Table II shows the reclassification, on a problem oriented basis, of young women who did not have STDs.

\section{LOWER GENITAL TRACT SYMPTOMS}

Patients complaining of lower genital tract symptoms, including vaginal discharge and pruritus vulvae, which were not attributable to candidal or trichomonal vaginitis or gonorrhoea, accounted for $14 \%(189 / 1373)$ in 1972 and $25 \%(448 / 1799)$ in 1982 of all those studied. No microbiological diagnosis was made in most patients in this group, and in 1972 such patients were either treated empirically with vaginal preparations and classified as having "other conditions requiring treatment" or were regarded as healthy and classified as having "other conditions

TABLE I No of diagnoses made in patients with sexually transmitted diseases and "other conditions" in 1972 and 1982

\begin{tabular}{lll}
\hline & \multicolumn{2}{l}{ No of girls studied in: } \\
\cline { 2 - 3 } & $\begin{array}{ll}1972 \\
(n=1373)\end{array}$ & $\begin{array}{l}1982 \\
(n=1799)\end{array}$ \\
\hline Sexually transmitted diseases & 1094 & 1008 \\
Other conditions & 613 & 1100 \\
\hline
\end{tabular}


TABLE II Reclassification of "other conditions" diagnosed in 1972 and 1982

\begin{tabular}{lcc}
\hline & \multicolumn{2}{c}{ No of girls studied in: } \\
\cline { 2 - 3 } \cline { 2 - 3 } Diagnosis & 1972 & 1982 \\
& $(n=1373)$ & $(n=1799)$ \\
\hline Lower genital tract symptoms & 189 & 448 \\
Upper genital tract symptoms & 5 & 22 \\
Urinary tract symptoms & 19 & 39 \\
Other gynaecological symptoms & 12 & 57 \\
Sexual contact with STD & 148 & 187 \\
Screening requests & 78 & 186 \\
Miscellaneous & 162 & 161 \\
Total & 613 & 1100 \\
\hline
\end{tabular}

not requiring treatment." By 1982 , bacterial vaginosis (non-specific vaginitis) was recognised as a separate entity and was diagnosed in 191 women.

UPPER GENITAL TRACT SYMPTOMS

Upper genital tract symptoms, which included abdominal pain and deep dyspareunia, were responsible for only five $(0.4 \%)$ attendances in 1972 and 22 $(1 \%)$ in 1982.

URINARY TRACT SYMPTOMS

Urinary tract symptoms, usually dysuria and frequency of micturition, were reported by 19 $(1 \cdot 4 \%)$ patients in 1972 and $39(2 \cdot 2 \%)$ patients in 1982.

\section{OTHER GYNAECOLOGICAL PROBLEMS}

The number of patients presenting with other gynaecological problems increased from $12(0.9 \%)$ in 1972 to $57(3 \cdot 2 \%)$ in 1982 . This increase was attributed to the number of young women in 1982 not classified elsewhere who were found to have abnormal cervical smears and who underwent colposcopy in the clinic. In 1972, such patients had been referred elsewhere.

\section{SEXUAL CONTACT WITH STD}

Epidemiological treatment of women who were sexual partners of men with gonorrhoea and nongonococcal urethritis (NGU) was clinic practice in both 1972 and 1982. Girls who were so treated comprised $10 \%$ of the study population in both years. In the case of those who were contacts of men with gonorrhoea, cultures for Neisseria gonorrhoeae gave negative results. In 1972 two patients had been given epidemiological treatment because they were sexual contacts of men with syphilis. No such cases occurred in 1982.

\section{SCREENING REQUEST}

The number of asymptomatic girls attending for screening for STDs increased from $78(6 \%)$ in 1972 to
$186(10 \%)$ in 1982 . No clinical or microbiological abnormality was detected in these young women.

\section{MISCELLANEOUS}

The remaining patients, $162(12 \%)$ in 1972 and 161 $(9 \%)$ in 1982, had problems which could not easily be classified. Some attended for counselling and others had conditions, such as tinea cruris, that were not classified elsewhere.

\section{Discussion}

A free and confidential service for diagnosing and treating venereal diseases, which are defined as syphilis, gonorrhoea, and chancroid, was established in the United Kingdom after the introduction of venereal diseases regulations in $1916 .{ }^{3}$ The number of patients with these diseases seen in STD clinics has been published yearly. After the second world war the number of cases of syphilis and gonorrhoea fell dramatically but the prevalence of other STDs began to increase in the 1950s. In 1951 NGU became notifiable for the first time, and in 1972 a major change in the reporting system was implemented with the introduction of seven new categories of disease, which included herpes simplex virus and genital warts.

By 1975, the value of reporting cases in the standard manner was being questioned ${ }^{4}$ and the importance of the category "other conditions" was stressed. More than one third of patients attending STD clinics with a wide variety of problems are classified in this way. Pattman and Schofield, who reviewed the yearly returns from a provincial clinic, suggested reclassifying patients in the groups "other conditions requiring, or not requiring, treatment". 5

The data from the study reported here confirm the national data and indicate that the largest increase in attendance is in the diagnostic category "other conditions". It is interesting that this observation applies as much to young girls as to the clinic population as a whole, as reports from the United States and comments in the media in this country have suggested that young girls are particularly at risk.

When a problem oriented approach was adopted, lower genital tract symptoms were found to be the most common to be classified within "other conditions". This remains an area of diagnostic confusion, no microbiological diagnosis being made in most cases. A culture service for Chlamydia trachomatis was not available in 1972 or in 1982, and some women with lower genital tract symptoms might have been infected with this micro-organism. It seems unlikely to have been responsible for the symptoms of many girls in this category, as previous studies have shown that most women infected with $C$ 
trachomatis are asymptomatic and the sexual partners of men with gonorrhoea or NGU. ${ }^{6}$ Even if such a diagnosis had been made, however, infected girls would still have been classified as having "another condition requiring treatment", as chlamydial infection is not a separate category in the annual return.

In 1982 a number of girls were diagnosed as having bacterial vaginosis (non-specific vaginitis). This condition should be accorded a separate diagnostic category when reproducible clinical and microbiological criteria are introduced for its diagnosis in the presence or absence of other STDs.

It is interesting to speculate why increasing numbers of women complaining of vaginal discharge should seek advice at an STD clinic. In the past, many women may have consulted general practitioners, but by 1982 were more willing to attend STD clinics where a microbiological diagnosis could be made. Furthermore, access to gynaecologists within the National Health Service is restricted by the length of waiting lists for non-urgent problems. Another possibility is that women with any gynaecological symptoms, for which they would previously have consulted a general practitioner or gynaecologist, may interpret them as being caused by an STD because a risk exists as a result of changing patterns of sexual behaviour. Further credence is given to the latter possibility by the large increase in numbers of asymptomatic women seeking screening for STDs. These possibilities should be investigated prospectively because of their imptications for the provision of health care.

It is clear that at present the reporting system is inadequate and does not reflect the changing pattern of STDs or the wide variety of clinical conditions seen. Though the classification described in this report is designed for the study of adolescent girls, a similar approach could be adopted for men that would include conditions such as hepatitis, bowel disorders, and the acquired immune deficiency syndrome.

\section{References}

1. Anonymous. Sexually transmitted disease surveillance, 1981. Br Med J 1983; 286: 1500-1.

2. Forster GE, Robinson GE, Munday PE. Sexually transmitted diseases: an epidemic in adolescent girls? British Journal of Venereal Diseases 1984;60:402-5.

3. Catterall RD. The British service for patients with sexually transmitted diseases. Health Trends 1983; 15:82-5.

4. Woodcock K. How useful are our present statistics on sexually transmitted diseases? British Journal of Venereal Diseases 1975;51: 153-7.

5. Pattman RS, Schofield CBS. Problem-orientated categorisation of "other conditions" seen in a genitourinary medicine clinic. British Journal of Venereal Diseases 1983; 59:63-5.

6. Munday PE. Chlamydial infections in women. In: J Studd, ed. Progress in obstetrics and gynaecology. Vol 3. London: Churchill Livingstone, 1983;231-45. 